Groups Geom. Dyn. 8 (2014), 553-564

DOI $10.4171 / \mathrm{GGD} / 238$
Groups, Geometry, and Dynamics

(C) European Mathematical Society

\title{
Endomorphisms of profinite groups
}

\author{
Colin D. Reid
}

\begin{abstract}
We obtain some general restrictions on the continuous endomorphisms of a profinite group $G$ under the assumption that $G$ has only finitely many open subgroups of each index (an assumption which automatically holds, for instance, if $G$ is finitely generated). In particular, given such a group $G$ and a continuous endomorphism $\phi$ we obtain a semidirect decomposition of $G$ into a 'contracting' normal subgroup and a complement on which $\phi$ induces an automorphism; both the normal subgroup and the complement are closed. If $G$ is isomorphic to a proper open subgroup of itself, we show that $G$ has an infinite abelian normal pro- $p$ subgroup for some prime $p$.
\end{abstract}

Mathematics Subject Classification (2010). $20 \mathrm{E} 18$.

Keywords. Profinite groups, endomorphisms of groups.

\section{Introduction}

Let $G$ be a finitely generated residually finite group, and let $\phi$ be an endomorphism. It is a well-known theorem of Mal'cev that $G$ is Hopfian, that is, if $\phi$ is surjective, then it is an isomorphism. On the other hand, there can certainly be injective endomorphisms of $G$ that are not surjective, such as the map $x \mapsto 2 x$ for $G=\mathbb{Z}$.

In this paper, we will focus on profinite groups, and all homomorphisms under consideration are understood to be continuous. Here the obvious analogue of 'finitely generated' is 'topologically finitely generated', but in fact it is appropriate to assume a somewhat weaker property:

Definition 1.1. Let $G$ be a profinite group. $G$ is of type (F) (or an (F)-group) if $G$ has finitely many open subgroups of index $n$ for every integer $n$. In other words, if we let $I_{n}^{\triangleleft}(G)$ be the intersection of all open normal subgroups of index at most $n$ in $G$, then $G$ is of type (F) if and only if $I_{n}^{\triangleleft}(G)$ is open in $G$ for all $n$.

Finitely-generated profinite groups are necessarily of type (F) (see for instance [4], Proposition 2.5.1.). In the other direction, a residually nilpotent profinite group $G$ is of type (F) if and only if its Sylow subgroups are all finitely generated, but this does 
not require any overall bound on the number of generators across different primes; thus one can easily construct examples of infinitely generated residually nilpotent (F)-groups. In addition, the class of (F)-groups includes all just infinite profinite groups, which need not be finitely generated even if they are hereditarily just infinite (see for instance [6]).

Profinite groups of type (F) are Hopfian as topological groups (see [4], Proposition 2.5.2.), but not co-Hopfian in general. Given a profinite group $G$, the question of whether $G$ has any proper open subgroups isomorphic to itself is particularly interesting in the theory of totally disconnected, locally compact groups. By a result of Willis ([5]), given a totally disconnected, locally compact group $L$ and a topological automorphism $\alpha$ of $G$, there is an open compact subgroup $U$ of $L$ such that $U=U_{+} U_{-}$, where $U_{+}=\bigcap_{i \geq 0} \alpha^{i}(U)$ and $U_{-}=\bigcap_{i \leq 0} \alpha^{i}(U)$. Thus $U_{+}$and $U_{-}$are profinite groups, with $\alpha\left(U_{-}\right)$an open subgroup of $U_{-}$and $U_{+}$an open subgroup of $\alpha\left(U_{+}\right)$, and we have $U_{-}=\alpha\left(U_{-}\right)$and $U_{+}=\alpha\left(U_{+}\right)$only in the case that $\alpha$ normalises $U$.

Given an endomorphism $\phi$ of a profinite group $G$, one can define two subgroups of $G$ which measure the extent to which positive powers of $\phi$ fail to be automorphisms:

$$
\operatorname{Con}(\phi)=\left\{x \in G \mid \lim _{n \rightarrow+\infty} \phi^{n}(x)=1\right\} ; \quad \phi_{+}(G)=\bigcap_{n \geq 0} \phi^{n}(G) .
$$

Example. Let $G=H \rtimes K$, where $H$ is the additive group of $\mathbb{Z}_{p}$ and $K$ is the group of units of $\mathbb{Z}_{p}$, acting by multiplication. Let $\phi$ be the endomorphism of $G$ that centralises $K$ and acts on $H$ as multiplication by $p$. Then $\operatorname{Con}(\phi)=H$ and $\phi_{+}(G)=K$.

Our first main result shows that this splitting as a semidirect product is a general phenomenon for profinite groups of type (F).

Theorem A. Let $G$ be a profinite group of type $(\mathrm{F})$, and let $\phi$ be an endomorphism of $G$. Then $G=\operatorname{Con}(\phi) \rtimes \phi_{+}(G)$ where $\operatorname{Con}(\phi)$ and $\phi_{+}(G)$ are both closed subgroups. The restriction of $\phi$ to $\phi_{+}(G)$ is an automorphism, and we have $\phi^{k}(\operatorname{Con}(\phi))=$ $\operatorname{Con}(\phi) \cap \phi^{k}(G)$ for all $k \geq 0$.

We now consider the possible structure of $\operatorname{Con}(\phi)$. Of course, any profinite group (of type (F)) can appear as $\operatorname{Con}(\phi)$ if $\phi$ is the zero endomorphism. More interesting is the case of open self-embeddings, that is, injective endomorphisms $\phi$ of $G$ whose image is open. In this case one has an associated ascending HNN extension $L=G *_{\phi}$ of $G$, and the topology of $G$ naturally extends to a group topology on $L$ with $G$ as an open subgroup. In effect, we are therefore considering special cases of the automorphisms and associated contraction groups as considered by Baumgartner, Glöckner and Willis in [1] and [2]. If the images of $G$ under the non-negative powers of $\phi$ have trivial intersection, then $G *_{\phi}$ is a contraction group in the sense of [2], which gives a good description of the structure of such groups. In the special case under consideration here, we can eliminate the case occurring in ([2], Theorem A) in which $G$ contains a Cartesian product of non-abelian finite simple groups. 
Definition 1.2. Given profinite groups $G$ and $H$, write $\operatorname{Hom}_{o}(G, H)$ for the set of injective homomorphisms from $G$ to $H$ with image open in $H$, and define $\operatorname{End}_{o}(G):=$ $\operatorname{Hom}_{o}(G, G)$. Given $\Lambda \subseteq \operatorname{End}_{o}(G)$, define

$$
O_{\Lambda}(G)=\overline{\langle\operatorname{Con}(\lambda) \mid \lambda \in \Lambda\rangle} .
$$

Theorem B. Let $G$ be a profinite group of type $(\mathrm{F})$, and let $\Lambda \subseteq \operatorname{End}_{o}(G)$.

(i) The group $O_{\Lambda}(G)$ is residually nilpotent.

(ii) Suppose that the set $\{\lambda(G) \mid \lambda \in \Lambda\}$ forms a base of neighbourhoods of the identity. Then $G=O_{\Lambda}(G)$, so $G$ is residually nilpotent.

When combined with Theorems A and B of [2], Theorem B (i) above can be refined to the following:

Corollary 1.3. Let $G$ be a profinite group of type (F), and let $\phi$ be an open selfembedding of $G$. Then $\operatorname{Con}(\phi)$ is an open subgroup of a direct product of finitely many uniform (in particular, torsion-free) pro- $p$ groups that are nilpotent and a residually nilpotent soluble group of finite exponent.

As a result we obtain the following, which generalises Theorem E (i) of [3].

Corollary 1.4. Let $G$ be a profinite group of type $(F)$. Suppose that there is a proper open subgroup of $G$ that is isomorphic to $G$ itself. Then there exists a prime number $p$ such that $G$ has an infinite abelian pro-p normal subgroup.

Theorems A and B will be proved in somewhat greater generality, requiring some more technical definitions to state. We will also prove some facts about open selfembeddings in the class of all profinite groups. At this point it seems reasonable to ask the following:

Question. Let $G$ be a finitely generated profinite group, and let $\phi$ be an open selfembedding of $G$. Is $\operatorname{Con}(\phi)$ necessarily nilpotent?

\section{Preliminaries and general remarks}

Given a set of sets $\mathcal{A}$, the expression $\bigcap \mathcal{A}\left(\right.$ respectively $\bigcup \mathcal{A}$ ) is used to mean $\bigcap_{A \in \mathscr{A}} A$ (respectively $\left.\bigcup_{A \in \mathcal{A}} A\right)$. The notation $H \leq_{o} G\left(H \unlhd_{o} G\right)$ means that $H$ is an open (normal) subgroup of $G$.

The following standard compactness argument will be used in several places.

Lemma 2.1. Let $G$ be a compact topological group, and let $O$ be an open neighbourhood of 1 in $G$. Let $\mathcal{K}$ be a set of closed subgroups of $G$ such that $\bigcap \mathcal{A} \nsubseteq O$ for every finite subset of of $\mathcal{K}$. Let $N=\bigcap \mathcal{K}$. Then $N \nsubseteq O$; in particular, $N$ is non-trivial. 
Proof. Given $K \in \mathcal{K}$, let $C_{K}=K \cap(G \backslash O)$. Then $\left\{C_{K} \mid K \in \mathcal{K}\right\}$ is a set of closed subsets of $G$ whose finite subsets have non-empty intersection. Since $G$ is compact, it follows that the intersection $N \cap(G \backslash O)$ of all the sets $C_{K}$ is non-empty. Hence $N \nsubseteq O$.

Corollary 2.2. Let $G$ be a profinite group, and let $H$ be a closed subgroup of $G$. Let $\mathcal{K}$ be a set of closed subgroups of $G$ such that $\overline{\langle\cap \mathcal{A}, H\rangle}=G$ for every finite subset $\mathcal{A}$ of $\mathcal{K}$. Let $K=\bigcap \mathcal{K}$. Then $\overline{\langle K, H\rangle}=G$.

Proof. Let $L=\overline{\langle K, H\rangle}$, and suppose $L<G$. Then $L \leq M$ for some proper open subgroup $M$ of $G$, since any closed subgroup of a profinite group is an intersection of open subgroups. Then $H \leq M$, and it follows from Lemma 2.1 that there is some finite subset $\mathcal{A}$ of $\mathcal{K}$ such that $\bigcap \mathcal{A} \leq M$. But then $\overline{\langle\bigcap \mathcal{A}, H\rangle} \leq M<G$, a contradiction.

The following trivial observation will be used without further comment.

Lemma 2.3. Let $G$ be a profinite group, let $K$ be a closed normal subgroup of $G$, and let $\phi$ be an endomorphism of $G$. Then $\phi^{-1}(K)$ is a closed normal subgroup of $G$.

When considering the existence of open embeddings between profinite groups, many questions reduce to those about profinite groups involving finitely many primes, thanks to the following:

Proposition 2.4. Let $G$ and $H$ be profinite groups, and suppose there is a continuous isomorphism $\phi$ from $G$ to an open subgroup $K$ of $H$. Given a set of primes $\pi$, write $O^{\pi}(G)$ for the intersection of all closed normal subgroups $L$ of $G$ such that $G / L$ is a pro- $\pi$ group. Then there is a finite set of primes $\pi$ such that the following holds for every set of primes $\pi^{*}$ containing $\pi$ :

There is an injective homomorphism $\psi$ from $G / O^{\pi^{*}}(G)$ to $H / O^{\pi^{*}}(H)$ such that $\psi \rho_{G}=\rho_{H} \phi$, where $\rho_{G}$ is the quotient map from $G$ to $G / O^{\pi^{*}}(G)$ and $\rho_{H}$ is the quotient map from $\mathrm{H}$ to $\mathrm{H} / \mathrm{O}^{\pi^{*}}(H)$.

Lemma 2.5. Let $G$ be a profinite group, and let $H$ be an open subgroup of $G$. Then there is a finite set of primes $\pi$ such that $O^{\pi^{*}}(H)=O^{\pi^{*}}(G)$, for any set of primes $\pi^{*}$ containing $\pi$.

Proof. Let $K$ be an open normal subgroup of $G$, let $\pi$ be the set of primes dividing $|G: K|$, and let $\pi^{*} \supseteq \pi$. Then $O^{\pi^{*}}(G) \leq K$ since $G / K$ is a $\pi$-group and hence a $\pi^{*}$ group. Moreover $O^{\pi^{*}}(K) \leq O^{\pi^{*}}(G)$ since $K / O^{\pi^{*}}(G)$ is contained in $G / O^{\pi^{*}}(G)$ and is therefore pro- $\pi^{*}$. On the other hand $G / O^{\pi^{*}}(K)$ is pro- $\pi^{*}$, being the extension of a pro- $\pi^{*}$ group by another pro- $\pi^{*}$ group, so in fact $O^{\pi^{*}}(K)=O^{\pi^{*}}(G)$.

We apply this argument inside $G$ and inside $H$ to obtain $O^{\pi^{*}}(G)=O^{\pi^{*}}(K)=$ $O^{\pi^{*}}(H)$ where $K$ is the core of $H$ in $G$, so the required finite set of primes $\pi$ is given by the prime divisors of $\left|G: \operatorname{Core}_{G}(H)\right|$. 
Proof of Proposition 2.4. By Lemma 2.5, there is a finite set of primes $\pi$ such that $O^{\pi^{*}}(\phi(G))=O^{\pi^{*}}(H)$, for any set of primes $\pi^{*}$ containing $\pi$. It follows that there is a well-defined and unique homomorphism $\psi$ from $G / O^{\pi^{*}}(G)$ to $H / O^{\pi^{*}}(H)$ such that

$$
\psi\left(x O^{\pi^{*}}(G)\right)=\phi(x) O^{\pi^{*}}(H)
$$

for all $x \in G$, in other words $\psi \rho_{G}=\rho_{H} \phi$. We also have $\psi\left(G / O^{\pi^{*}}(G)\right)=$ $\phi(G) O^{\pi^{*}}(H) \leq_{o} H$, and

$$
\operatorname{ker}(\psi)=\left\{x O^{\pi^{*}}(G) \mid x \in G, \phi(x) \in O^{\pi^{*}}(\phi(G))\right\}=1 .
$$

Hence $\psi$ is injective.

Preimages of endomorphisms are well-behaved with respect to indices of open subgroups.

Lemma 2.6. Let $G$ be a profinite group, let $K$ be an open subgroup of $G$, and let $\phi$ be an endomorphism of $G$. Then $\left|G: \phi^{-1}(K)\right| \leq|G: K|$. If $\left|G: \phi^{-1}(K)\right|=|G: K|$ then $G=\phi(G) K$.

Proof. Note that $|\phi(G): K \cap \phi(G)| \leq|G: K|$, with equality if and only if $\phi(G)$ contains a transversal of $K$ in $G$, that is, if and only if $G=\phi(G) K$. Note also that $\phi$ induces a surjective map from left cosets of $L$ in $G$ to left cosets of $K \cap \phi(G)$ in $\phi(G)$, where $L=\phi^{-1}(K)$. Suppose $|G: L|>|\phi(G): K \cap \phi(G)|$. Then by the pigeon-hole principle, there are distinct cosets $x L$ and $y L$ of $L$ in $G$ such that $\phi(x)(K \cap \phi(G))=\phi(y)(K \cap \phi(G))$. But then $\phi\left(x y^{-1}\right)=\phi(x) \phi\left(y^{-1}\right) \in K$, so in fact $x y^{-1} \in L$, a contradiction. Hence $|G: L| \leq|\phi(G): K \cap \phi(G)| \leq|G: K|$, and if $|G: L|=|G: K|$ then $G=\phi(G) K$.

To determine whether every open subgroup of a profinite group $G$ contains an open subgroup isomorphic to $G$, it suffices to consider maximal open normal subgroups.

Proposition 2.7. Let $G$ be a profinite group, and let $H$ be an open subgroup of $G$. Suppose that $\operatorname{Hom}_{o}(G, H)=\emptyset$. Then $\operatorname{Hom}_{o}(G, K)=\emptyset$ for some open normal subgroup $K$ of $G$ such that $G / K$ is simple.

Proof. By replacing $H$ by its core in $G$, we may assume $H$ is normal in $G$; moreover, we may assume $|G: H|$ realises the minimum value of the set

$$
\left\{|G: N| \mid N \unlhd_{o} G, \operatorname{Hom}_{o}(G, N)=\emptyset\right\} .
$$

Clearly $H<G$, so there is a normal subgroup $K$ containing $H$ such that $G / K$ is simple. Suppose there exists $\phi \in \operatorname{Hom}_{o}(G, K)$; let $L=\phi^{-1}(H)$. Then $\phi(G) H \leq$ $K<G$, so $|G: L|<|G: H|$ by Lemma 2.6. By the minimality of $|G: H|$, there must be some $\psi \in \operatorname{Hom}_{o}(G, L)$. But then $\left.\phi\right|_{L} \psi \in \operatorname{Hom}_{o}(G, H)$, a contradiction. Hence $\operatorname{Hom}_{o}(G, K)=\emptyset$. 
As an extreme case, one could consider profinite groups $G$ in which every open subgroup $H$ of $G$ is isomorphic to $G$, or at least there is some (open) normal subgroup of $G$ contained in $H$ that is isomorphic to $G$. However, there are no examples of this behaviour for groups of type $(\mathrm{F})$ aside from the obvious ones.

Proposition 2.8. Let $G$ be a profinite group of type (F). Then the following are equivalent:

(i) Every open subgroup of $G$ contains a normal subgroup $N$ of $G$ such that $N \cong G$.

(ii) $G$ is of the form $\prod_{p} \mathbb{Z}_{p}^{n_{p}}$ for non-negative integers $n_{p}$, where $p$ ranges over $a$ set of prime numbers. In particular, every open subgroup of $G$ is isomorphic to $G$.

Proof. It is clear that (ii) implies (i). Suppose (i) holds; let $\ell=\left\{I_{n}^{\triangleleft}(G) \mid n \in \mathbb{N}\right\}$.

We claim first that $G$ is abelian. Since $\ell$ forms a base of neighbourhoods of the identity, it suffices to show that $G / H$ is abelian for all $H \in \bigodot$. Fix $H$; let $k=|\operatorname{Aut}(G / H)|$, and let $K=I_{k}^{\triangleleft}(G)$. Then by our assumption, there is an injective endomorphism $\phi$ of $G$ such that $\phi(G)$ is normal in $G$ and $\phi(G) \leq K$. Since $\phi(H)$ is a characteristic subgroup of $\phi(G)$, we see that $G$ acts on $\phi(G) / \phi(H)$ by conjugation as a subgroup of $\operatorname{Aut}(\phi(G) / \phi(H)) \cong \operatorname{Aut}(G / H)$, so $\left|G: C_{G}(\phi(G) / \phi(H))\right| \leq K$, and hence $K \leq C_{G}(\phi(G) / \phi(H))$. In particular, $\phi(G)$ acts trivially on $\phi(G) / \phi(H)$, so $\phi(G) / \phi(H)$ is abelian, and thus $G / H$ is abelian.

Thus $G$ is a Cartesian product of its Sylow subgroups $S_{p}$, each of which is abelian of type (F). This forces $S_{p}=F_{p} \times \mathbb{Z}_{p}^{n_{p}}$ where $F_{p}$ is finite; in fact $F_{p}$ must be trivial for (i) to hold, by considering the $p$-torsion subgroups of open subgroups of $G$. Thus $G$ is of the form described in (ii).

\section{Well-behaved groups and endomorphisms}

We now define a somewhat technical property of a set of endomorphisms acting on a profinite group. This property holds for all endomorphisms of (F)-groups, but is additionally retained on passage to suitable invariant subgroups.

Definition 3.1. Let $G$ be a profinite group, and let $\Lambda$ be a set of endomorphisms of $G$. A subgroup $H$ of $G$ is $\Lambda$-invariant if $\lambda(H) \leq H$ for all $\lambda \in \Lambda$. We define $\Lambda$ to be stable on $G$ if the set of all $\Lambda$-invariant open subgroups of $G$ is a base of neighbourhoods of the identity.

Given a set $\Omega$ of automorphisms of $G$, let $I_{n}^{\Omega}(G)$ be the intersection of all $\Omega$ invariant open normal subgroups of $G$ of index at most $n$. We will say $\Omega$ regulates $\Lambda$ (on $G$ ) if the following conditions are satisfied:

(a) $\lambda \Omega=\Omega \lambda$ for all $\lambda \in \Lambda$. 
(b) $I_{n}^{\Omega}(G)$ is open in $G$ for all $n$.

(c) The set $\left\{I_{n}^{\Omega}(G) \mid n \in \mathbb{N}\right\}$ is a base of neighbourhoods of the identity.

We define $\Lambda$ to be regulated on $G$ if a set of $\Omega$ of automorphisms exists such that $\Omega$ regulates $\Lambda$. Note that subsets of regulated sets are regulated.

If $G$ is of type (F) then the set of all endomorphisms is regulated (for instance by the empty set).

Proposition 3.2. Let $G$ be a profinite group, and let $\Lambda$ be a set of endomorphisms of $G$. Suppose that $\Lambda$ is regulated by $\Omega \subseteq \operatorname{Aut}(G)$ on $G$.

(i) If $K$ is an $\Omega$-invariant open normal subgroup of $G$ and $\lambda \in \Lambda$, then $\lambda^{-1}(K)$ is an $\Omega$-invariant open normal subgroup of $G$. As a result, $I_{n}^{\Omega(}(G)$ is $\Lambda$-invariant for all $n$, so $\Lambda$ is stable on $G$.

(ii) Suppose that $G$ is of the form $N \rtimes H$ where both $N$ and $H$ are $\Lambda \cup \Omega$-invariant, and where every element of $\Lambda$ induces a surjective map on $H$. Let $\Psi$ be the set of automorphisms of $N$ induced by the conjugation action of $H$. Then $\Xi=\Omega \cup \Psi$ regulates $\Lambda$ acting on $N$.

Proof. (i) Let $\lambda \in \Lambda$ and $\omega \in \Omega$. Then $\lambda \omega=\omega^{\prime} \lambda$ for some $\omega^{\prime} \in \Omega$, so

$$
\lambda \omega\left(\lambda^{-1}(K)\right)=\omega^{\prime} \lambda\left(\lambda^{-1}(K)\right)=\omega^{\prime}(K \cap \lambda(G)) \leq \omega^{\prime}(K)=K
$$

and thus $\omega\left(\lambda^{-1}(K)\right) \leq \lambda^{-1}(K)$, so $\lambda^{-1}(K)$ is $\Omega$-invariant. We have $\left|G: \lambda^{-1}(K)\right| \leq$ $|G: K|$ by Lemma 2.6 , so $\lambda^{-1}(K)$ is open in $G$. It follows that $\lambda^{-1}\left(I_{n}^{\Omega}(G)\right)$ is an intersection of $\Omega$-invariant open normal subgroups of $G$ of index at most $n$, so $\lambda^{-1}\left(I_{n}^{\Omega}(G)\right) \geq I_{n}^{\Omega}(G)$, that is $\lambda\left(I_{n}^{\Omega}(G)\right) \leq I_{n}^{\Omega}(G)$.

(ii) We must check that each of the conditions (a)-(c) are satisfied by $\Xi$.

Let $\lambda \in \Lambda$. We have $\lambda \Omega=\Omega \lambda$ by assumption. Suppose $h \in H$ induces $\psi_{h} \in \Psi$ by conjugation. Then for all $x \in N$ :

$$
\lambda \psi_{h}(x)=\lambda\left(h x h^{-1}\right)=\lambda(h) \lambda(x) \lambda(h)^{-1}=\psi_{\lambda(h)} \lambda(x),
$$

so $\lambda \psi_{h}=\psi_{\lambda(h)} \lambda$. Similarly $\psi_{h} \lambda=\lambda \psi_{k}$, where $k$ is an element of $H$ such that $\lambda(k)=h$. This proves condition (a).

Let $K$ be a $\Xi$-invariant open normal subgroup of $N$ of index at most $n$. Then $K$ is normalised by $H$, so $K \unlhd G$, and $K H$ is an open $\Omega$-invariant subgroup of $G$, so the core of $K H$ in $G$ is an open normal $\Omega$-invariant subgroup of $G$. Thus $K H \geq I_{n !}^{\Omega}(G)$, which ensures that $K$ contains $I_{n !}^{\Omega}(G) \cap N$. Thus $I_{n}^{\Xi}(N)$ contains the open subgroup $I_{n !}^{\Omega(}(G) \cap N$ of $N$. Moreover, we see that $I_{t(n)}^{\Xi}(N) \leq I_{n}^{\Omega}(G) \cap N$ for all $n \in \mathbb{N}$, where $t(n)=\left|G: I_{n}^{\Omega}(G)\right|$, so the set $\left\{I_{n}^{\Xi}(N) \mid n \in \mathbb{N}\right\}$ has trivial intersection. This proves conditions (b) and (c). 
We can also generalise the hypotheses of Theorem A by considering contraction for more general semigroups of endomorphisms, in place of the cyclic monoid $\left\{\phi^{k} \mid\right.$ $k \geq 0\}$.

Definition 3.3. Let $G$ be a profinite group, and let $\Lambda$ be a semigroup of endomorphisms of $G$ acting on the left. Write $\Lambda_{\cap}(G)$ for $\bigcap_{\lambda \in \Lambda} \lambda(G)$. Let $\mathcal{F}$ consist of all subsets of $\Lambda$ which contain $\bigcap_{\xi \in \Xi} \Lambda \xi$ for some finite subset $\Xi$ of $\Lambda$. Given a closed subgroup $K$ of $G$, let $\operatorname{Con}(\Lambda, K)$ be the set of elements $x \in G$ such that for all open sets $O \supseteq K$ there is some $\Sigma \in \mathcal{F}$ such that $\sigma x \in O$ for all $\sigma \in \Sigma$; define $\operatorname{Con}(\Lambda)=\operatorname{Con}(\Lambda, 1)$. (One can regard $\operatorname{Con}(\Lambda)$ as the set on which $\Lambda$ converges pointwise to the zero endomorphism.) For these definitions to be useful, we are particularly interested in those semigroups $\Lambda$ which satisfy following conditions:

(I) For every finite subset $\Xi$ of $\Lambda$, the intersection $\bigcap_{\xi \in \Xi} \Lambda \xi$ is non-empty.

(II) For every finite subset $\Xi$ of $\Lambda$, there is some $\lambda \in \Lambda$ such that $\lambda(G) \leq \Xi_{\cap}(G)$.

Notice that conditions (I) and (II) are automatically satisfied if $\Lambda$ is a commutative semigroup. In the simplest case, when $\Lambda$ is the set of non-negative powers of a single endomorphism $\phi$, we see that $\operatorname{Con}(\Lambda)=\operatorname{Con}(\phi)$ and $\Lambda_{\cap}(G)=\phi_{+}(G)$.

Lemma 3.4. Let $G$ be a profinite group, and let $\Lambda$ be a semigroup of endomorphisms of $G$. Let $K$ be a closed subgroup of $G$.

(i) The set $\operatorname{Con}(\Lambda, K)$ is a subgroup of $G$, and if $K \unlhd G$ then $\operatorname{Con}(\Lambda, K) \unlhd G$.

(ii) Given $\lambda \in \Lambda$ and $x \in G$, then $\lambda(\operatorname{Con}(\Lambda, K)) \geq \operatorname{Con}(\Lambda, K) \cap \lambda(G)$ and $\operatorname{ker} \lambda \leq \operatorname{Con}(\Lambda, K)$.

Proof. (i) Since we have

$$
\operatorname{Con}(\Lambda, K)=\bigcap_{L \in \mathscr{L}} \operatorname{Con}(\Lambda, L),
$$

where $\mathscr{L}$ is any set of open subgroups of $G$ with intersection $K$, we may assume that $K$ is open in $G$.

Given $x, y \in \operatorname{Con}(\Lambda, K)$, there exist $\Sigma_{1}, \Sigma_{2} \in \mathcal{F}$ such that

$$
x \in \bigcap_{\sigma \in \Sigma_{1}} \sigma^{-1}(K) \text { and } y \in \bigcap_{\sigma \in \Sigma_{2}} \sigma^{-1}(K) .
$$

Since $\mathcal{F}$ is a filter we also have $\Sigma_{3}=\Sigma_{1} \cap \Sigma_{2} \in \mathcal{F}$, and since the preimages $\sigma^{-1}(K)$ are always subgroups of $G$, it follows that $x y^{-1} \in \bigcap_{\sigma \in \Sigma_{3}} \sigma^{-1}(K) \subseteq \operatorname{Con}(\Lambda, K)$. Hence $\operatorname{Con}(\Lambda, K)$ is a group.

Now suppose $K \unlhd G$ and let $x \in \operatorname{Con}(\Lambda, K)$. Then there is some $\Sigma \in \mathcal{F}$ such that $\sigma(x) \in K$ for all $\sigma \in \Sigma$. This implies that $\sigma\left(y^{-1} x y\right) \in K$ for all $\sigma \in \Sigma$ and $y \in G$.

(ii) Let $x \in G$, and let $y=\lambda(x)$; suppose $y \in \operatorname{Con}(\Lambda, K)$. Then for all open sets $O$ containing $K$, there is some $\Sigma \in \mathcal{F}$ such that $\sigma \lambda(x)=\sigma(y) \in O$ for all $\sigma \in \Sigma$. Since $\Sigma \lambda \in \mathscr{F}$ it follows that $x \in \operatorname{Con}(\Lambda, K)$. The case $y=1$ shows that ker $\lambda \leq$ $\operatorname{Con}(\Lambda, K)$, and in general we see that $\lambda(\operatorname{Con}(\Lambda, K)) \geq \operatorname{Con}(\Lambda, K) \cap \lambda(G)$. 


\section{Main theorems}

Theorem 4.1. Let $G$ be a countably based profinite group. Let $\Lambda$ be a semigroup of endomorphisms of $G$ that is stable and satisfies condition (I). Then $\operatorname{Con}(\Lambda)$ is a closed normal subgroup of $G$ that has trivial intersection with $\Lambda_{\cap}(G)$. For any $\lambda \in \Lambda$ we have $G=\operatorname{Con}(\Lambda) \lambda(G)$ and $\operatorname{Con}(\Lambda) \cap \lambda(G)=\lambda(\operatorname{Con}(\Lambda))$. In particular, $\Lambda$ restricts to a stable semigroup of endomorphisms of $\operatorname{Con}(\Lambda)$ and $\Lambda_{\cap}(\operatorname{Con}(\Lambda))=1$.

If in addition $\Lambda$ satisfies condition (II), then $G=\operatorname{Con}(\Lambda) \rtimes \Lambda_{\cap}(G)$ and every $\lambda \in \Lambda$ restricts to an automorphism on $\Lambda_{\cap}(G)$.

Proof. Let $N=\operatorname{Con}(\Lambda)$ and $H=\Lambda_{\cap}(G)$.

Let $K$ be an open subgroup of $G$. For $\Sigma \in \mathcal{F}$ write $R_{\Sigma}=\bigcap_{\sigma \in \Sigma} \sigma^{-1}(K)$, and let $\mathcal{R}=\left\{R_{\Sigma} \mid \Sigma \in \mathscr{F}\right\}$. Then $K$ contains a $\Lambda$-invariant open subgroup $L$ of $G$, so $R_{\Sigma} \geq L$ for all $\Sigma \in \mathscr{F}$. Hence $\operatorname{Con}(\Lambda, K)$ is an open subgroup of $G$, since $\operatorname{Con}(\Lambda, K)=\bigcup \mathcal{R}$. Thus by Lemma 3.4, we have

$$
\begin{aligned}
|G: \operatorname{Con}(\Lambda, K)| & \geq|\lambda(G): \lambda(G) \cap \operatorname{Con}(\Lambda, K)| \\
& \geq|\lambda(G): \lambda(\operatorname{Con}(\Lambda, K))|=|G: \operatorname{Con}(\Lambda, K)|,
\end{aligned}
$$

where all indices are finite. We see that in fact equality must hold for both inequalities here.

For the first inequality, this ensures $\operatorname{Con}(\Lambda, K) \lambda(G)=G$. We let $K$ range over a descending chain of open subgroups with trivial intersection; the corresponding groups $\operatorname{Con}(\Lambda, K)$ then also form a descending chain. Applying Corollary 2.2 then gives $N \lambda(G)=G$.

Given Lemma 3.4 (ii), the equality $|\lambda(G): \lambda(G) \cap \operatorname{Con}(\Lambda, K)|=\mid \lambda(G)$ : $\lambda(\operatorname{Con}(\Lambda, K)) \mid$ implies that $\operatorname{Con}(\Lambda, K) \cap \lambda(G)=\lambda(\operatorname{Con}(\Lambda, K))$ for all open subgroups $K$; this implies that $N \cap \lambda(G)=\lambda(N)$, so $N \cap H=\Lambda_{\cap}(N)$.

We have $\operatorname{Con}(\Lambda, K)=\bigcup \mathcal{R}$; in fact, $\mathcal{R}$ is a direct system of open subgroups, since $R_{\Sigma_{1} \cap \Sigma_{2}} \geq\left\langle R_{\Sigma_{1}}, R_{\Sigma_{2}}\right\rangle$, so by the compactness of $\operatorname{Con}(\Lambda, K)$ we must have $\operatorname{Con}(\Lambda, K)=R_{\Sigma}$ for some $\Sigma$ depending on $K$. Condition (I) ensures this set $\Sigma$ is non-empty, in other words there exists $\sigma \in \Lambda$ such that $\sigma(\operatorname{Con}(\Lambda, K)) \leq K$, so $\Lambda_{\cap}(\operatorname{Con}(\Lambda, K)) \leq K$. Since $N$ is the intersection of $\operatorname{Con}(\Lambda, K)$ as $K$ ranges over the open subgroups, it follows that $N$ is closed and $\Lambda_{\cap}(N)=1$, and hence $N \cap H=1$.

Now suppose condition (II) holds. Then we have $N \Xi_{\cap}(G)=G$ for all finite subsets $\Xi$ of $\Lambda$, so $G=N H$ by Corollary 2.2 and hence $G=N \rtimes H$.

Fix $\lambda \in \Lambda$; it remains to show that $\lambda$ induces an automorphism on $H$. We have $H \cap \operatorname{ker} \lambda \leq H \cap N=1$, so $\lambda$ induces an isomorphism from $H$ to $\lambda(H)$; since $\lambda \Lambda \subseteq \Lambda$, we also have $\lambda(H) \geq H$. If $\lambda(H)>H$, then since $G=N H$ we must have some $x \in H \backslash\{1\}$ such that $\lambda(x) \in N$. But then $x \in N$ as in the proof of Lemma 3.4 (ii), so $x \in N \cap H=1$, a contradiction. 
Theorem 4.1 and Proposition 3.2 combined imply Theorem A. They also have the following consequence:

Corollary 4.2. Let $G$ be a countably based profinite group. Let $\Lambda$ be a semigroup of endomorphisms of $G$ that is regulated and satisfies conditions (I) and (II). Then $\Lambda$ is regulated on $\operatorname{Con}(\Lambda)$.

The following theorem is a more general form of Theorem B. Corollary 1.3 can of course be generalised in the same way.

Theorem 4.3. Let $G$ be a profinite group, and let $\Lambda \subseteq \operatorname{End}_{o}(G)$. Suppose that $\Lambda$ is regulated on $G$.

(i) The group $O_{\Lambda}(G)$ is residually nilpotent.

(ii) Suppose that the set $\{\lambda(G) \mid \lambda \in \Lambda\}$ forms a base of neighbourhoods of the identity. Then $G=O_{\Lambda}(G)$, so $G$ is residually nilpotent.

Proof. (i) To show that $O_{\Lambda}(G)$ is residually nilpotent, it suffices to show $\operatorname{Con}(\lambda)$ is residually nilpotent for all $\lambda \in \Lambda$, since any profinite group has a unique largest residually nilpotent normal subgroup. Note that the set $\Delta$ of non-negative powers of $\lambda$ is regulated on $G$ and hence also on $\operatorname{Con}(\lambda)$ by Corollary 4.2. Thus we may assume $G=\operatorname{Con}(\lambda)$.

Let $k=|G: \lambda(G)|$; we may assume $k>1$, as otherwise $\lambda$ is an automorphism and $\operatorname{Con}(\lambda)=1$. Let $\Omega$ be a regulating set for $\Delta$ on $G$, and equip $\Omega$ with the discrete topology. Define the following series of subgroups of $L=G \rtimes \Omega$ :

$$
N_{0}=G ; \quad N_{i+1}=N_{i} \cap \operatorname{Core}_{N_{i} \Omega}\left(\left(\lambda^{i+1}(G) \cap N_{i}\right) \Omega\right) .
$$

Let $i \in \mathbb{N}$. By construction, $N_{i}$ is $\Omega$-invariant and we have $N_{i} \leq \lambda^{i}(G)$ (so in particular $\left.\bigcap N_{i}=1\right)$ and $N_{i+1} \unlhd N_{i}$.

Given $\omega \in \Omega$, note that $\omega \lambda^{i+1}=\lambda^{i+1} \omega^{\prime}$ for some $\omega^{\prime} \in \Omega$ (as endomorphisms of $G$ ) by condition (a). Thus $\lambda^{i+1}(G) \cap N_{i}$ is normalised by $\Omega$ in $L, \operatorname{so}\left(\lambda^{i+1}(G) \cap N_{i}\right) \Omega$ is a subgroup of $N_{i} \Omega$ of index at most $k$, and hence

$$
\left|N_{i}: N_{i+1}\right|=\left|N_{i} \Omega: N_{i+1} \Omega\right| \leq k !
$$

Now let $M$ be an open subgroup of $G$ that is maximal subject to the conditions that $M$ is $\Omega$-invariant and that there is an $\Omega$-invariant closed subnormal series from $M$ to $L$; let $\Phi^{\Omega}(G)$ be the intersection of all such subgroups $M$. Then clearly $M \triangleleft G$, so $M$ is a maximal $\Omega$-invariant open normal subgroup of $G$. As $M$ is a proper open subgroup of $G$, there is some $i$ such that $N_{i} \not \leq M$ but $N_{i+1} \leq M$. Thus $M N_{i}$ is $\Omega$-invariant, and there is an $\Omega$-invariant subnormal series from $M N_{i}$ to $G$; thus $M N_{i}=G$ by the maximality of $M$. This ensures that $|G: M|=\mid M N_{i}$ : $M N_{i+1}|\leq| N_{i}: N_{i+1} \mid \leq k$ !. It follows that $\Phi^{\Omega}(G) \geq I_{k !}^{\Omega}(G)$, so $\Phi^{\Omega}(G)$ is an open 
subgroup of $G$. Thus there exists $i$ such that $\lambda^{i}(G) \leq \Phi^{\Omega}(G)$, since $G=\operatorname{Con}(\lambda)$; by replacing $\lambda$ with $\lambda^{i}$ we may assume $i=1$.

Let $H=\lambda(G)$. Given $t \in \mathbb{N}$, let $G_{t}=I_{t}^{\Omega}(G)$ and $H_{t}=I_{t}^{\Omega}(H)$. Consider a proper $\Omega$-invariant open normal subgroup $K$ of $G$ such that $|G: K| \leq t+1$. Then $K H$ is a proper open subgroup of $G$, since it is contained in $K \Phi^{\Omega}(G)$. In addition, $H \cap K$ is an $\Omega$-invariant open normal subgroup of $H$, and $|H: H \cap K|=\mid K H$ : $K|<| G: K \mid$; in particular, $|H: H \cap K| \leq t$. Hence $K$ contains $H_{t}$. Since this argument holds for all $t$, and for all proper $\Omega$-invariant open normal subgroups of $G$ of index at most $t+1$, it follows that $G_{t+1} \geq H_{t}$ for all $t$. Hence:

$$
\left|G: H_{t}\right|=k\left|H: H_{t}\right|=\left|G: G_{t}\right|\left|G_{t}: G_{t+1}\right|\left|G_{t+1}: H_{t}\right| .
$$

Now $\lambda$ induces an isomorphism from $G$ to $H$ that sends $\Omega$-invariant subgroups to $\Omega$-invariant subgroups (by virtue of condition (a) in Definition 3.1), accounting in this way for all $\Omega$-invariant subgroups of $H$. Hence $\left|H: H_{t}\right|=\left|G: G_{t}\right|$, so $\left|G_{t}: G_{t+1}\right|$ divides $k$ for all $t$.

Let $n$ be the largest order of $\operatorname{Aut}(F)$, as $F$ ranges over all finite groups of order dividing $k$, and let $R=I_{n}^{\Omega}(G)$. Then for all $t$, the centraliser of $G_{t} / G_{t+1}$ in $G$ is an $\Omega$-invariant open normal subgroup of $G$ of index at most $n$, and so contains $R$; thus the series $\left(R \cap G_{t}\right)_{t \in \mathbb{N}}$ is a descending central series for $R$, and in particular $R$ is residually nilpotent. Moreover $R$ has finite index in $G$, so there is some $i$ such that $\lambda^{i}(G) \leq R$; since $G \cong \lambda^{i}(G)$, it follows that $G$ is residually nilpotent.

(ii) Suppose $O_{\Lambda}(G)<G$. Then there is some proper open subgroup $K$ of $G$ containing $O_{\Lambda}(G)$, and some $\lambda \in \Lambda$ such that $\lambda(G) \leq K$ and hence $O_{\Lambda}(G) \lambda(G) \leq$ $K$; in particular, $\operatorname{Con}(\lambda) \lambda(G) \leq K$. However, by Proposition 3.2 and Theorem 4.1 we have $\operatorname{Con}(\lambda) \lambda(G)=G$, a contradiction.

Acknowledgements. My thanks go to Charles Leedham-Green for helpful discussions on an earlier version of this paper.

\section{References}

[1] U. Baumgartner and G. A. Willis, Contraction groups and scales of automorphisms of totally disconnected locally compact groups. Israel J. Math. 142 (2004), 221-248. Zbl 1056.22001 MR 2085717

[2] H. Glöckner and G. A. Willis, Classification of the simple factors appearing in composition series of totally disconnected contraction groups. J. Reine Angew. Math. 643 (2010), 141-169. Zbl 1196.22005 MR 2658192

[3] C. D. Reid, On the structure of just infinite profinite groups. J. Algebra 324 (2010), 2249-2261. Zbl 1213.20027 MR 2684140

[4] L. Ribes and P. Zalesskii, Profinite groups. Ergeb. Math. Grenzgeb. (3) 40, Springer, Berlin 2000. Zbl 1197.20022 MR 1775104 
[5] G. A. Willis, The structure of totally disconnected, locally compact groups. Math. Ann. 300 (1994), no. 2, 341-363. Zbl 0811.22004 MR 1299067

[6] J. S. Wilson, Large hereditarily just infinite groups. J. Algebra 324 (2010), no. 2, 248-255. Zbl 1209.20028 MR 2651355

Received April 10, 2012; revised February 21, 2013

C. D. Reid, University of Newcastle, School of Mathematical and Physical Sciences, University Drive, Callaghan NSW 2308, Australia

E-mail: colin@ reidit.net 